\title{
Impact of COVID-19 in emergency medicine literature: a bibliometric analysis
}

\author{
Marco Vitolo ${ }^{1,2} \cdot$ Andrea Venturelli $^{1} \cdot$ Anna Chiara Valenti ${ }^{1} \cdot$ Giuseppe Boriani ${ }^{1}$ (i)
}

Received: 11 November 2021 / Accepted: 27 November 2021 / Published online: 17 January 2022

(c) The Author(s), under exclusive licence to Società Italiana di Medicina Interna (SIMI) 2021

Keywords COVID-19 · Pandemic · Emergency medicine · SARS-CoV2 $\cdot$ Coronavirus · Literature · Medical journals

Dear Editor,

Since the first cases of coronavirus disease (COVID-19) in December 2019, a growing amount of scientific articles on this topic have been published [1]. It has been reported that around $4 \%$ of the world's research output in 2020 was related to COVID-19 [2, 3]

During the COVID-19 pandemic, the researcher community had to promptly produce valid and rigorous scientific data to support new treatments, healthcare reorganizations and to investigate the pathophysiological background of the disease $[4,5]$. The rapid dissemination of the disease forced scientific journals to reduce the median time to the publication of COVID-19 related articles and the peer-review process was significantly shortened, limiting in some cases their scientific quality [6]. The ongoing COVID-19 dataset from Dimensions.ai (including all published articles, preprints, clinical trials related to COVID-19 from a variety of sources including Crossref, PubMed, Europe PubMed Central, arXiv and direct contacts with more than 130 publishers) reports more than 690,000 publications involving 29,000 organizations and 199 countries (https://reports.dimensions.ai/covid19/. Accessed Nov 2021).

Recently, different bibliometric analyses have been published focusing on the impact of COVID-19 on medical literature [3]. However, although emergency physicians are primarily involved in the pandemic, specific analyses on the

Giuseppe Boriani

giuseppe.boriani@unimore.it

1 Cardiology Division, Department of Biomedical, Metabolic and Neural Sciences, University of Modena and Reggio Emilia, Policlinico di Modena, Via del Pozzo, 71, 41124 Modena, Italy

2 Clinical and Experimental Medicine PhD Program, University of Modena and Reggio Emilia, Modena, Italy contribution of emergency medicine (EM) journals to the COVID-19 literature are limited.

Accordingly, we aimed to assess the proportion of COVID-19 related papers published in EM journals with a specific focus on the most discussed topics within a sample of EM journals.

We conducted a review of scientific papers regarding COVID-19 published from 1st January 2020 to 31 st May 2021 in ten top-ranked emergency medicine journals (Table 1). First, we retrieved from PubMed the total number of publications in the ten journals during the selected period. Subsequently, we performed a systematic search through PubMed using the following keywords: "Sars-cov 2", "COVID", "COVID-19" and their related terms in the same journals and for the same period. Two investigators independently (MV and AV) screened records for eligibility based on title and abstract. Disagreements during the screening phase were discussed and resolved by a third reviewer (ACV). One senior reviewer (GB) analyzed the study selection and the data extraction process. We analyzed the proportion of COVID-19-related papers on the total number of scientific papers published. Selected articles on COVID-19 were categorized based on their type (Fig. 1). Corrigendum and retracted articles were excluded. Finally, we analyzed the absolute number of the main topics related to COVID-19 as considered by published papers among the ten selected journals (Fig. 2).

The total number of scientific papers published in the ten journals during the specified period of time was 4954 . After the exclusion of 17 papers (of which ten corrigendum, six not pertinent articles, and one duplicate), we included in the analysis a total of 929 COVID-related articles (19\% of the total) (Table 1). There was some heterogeneity with regard to the proportion of COVID-related articles varied from the minimum of 5\% in the World Journal of Emergency 
Table 1 Total number and proportion of COVID-19 related papers published during the study period

\begin{tabular}{lllll}
\hline & $\begin{array}{l}\text { Impact Factor } \\
\text { Year 2020* }\end{array}$ & $\begin{array}{l}\text { Total Pub- } \\
\text { lications }\end{array}$ & $\begin{array}{l}\text { COVID-19 } \\
\text { Publications }\end{array}$ & $\begin{array}{l}\text { COVID- } \\
19 / \text { total } \\
(\%)\end{array}$ \\
\hline Annals of Emergency Medicine & 5.72 & 572 & 63 & 11 \\
Academic Emergency Medicine & 3.45 & 414 & 59 & 14 \\
Internal and Emergency Medicine & 3.39 & 607 & 174 & 29 \\
European Journal of Emergency Medicine & 2.79 & 192 & 21 & 11 \\
Emergency Medicine Journal & 2.74 & 383 & 77 & 20 \\
American Journal of Emergency Medicine & 2.46 & 1827 & 369 & 20 \\
Western Journal of Emergency Medicine & 2.45 & 346 & 92 & 27 \\
Canadian Journal of Emergency Medicine & 2.41 & 336 & 56 & 17 \\
World Journal of Emergency Medicine & 2.26 & 115 & 6 & 5 \\
BMC Emergency Medicine & 2.11 & 162 & 12 & 7 \\
Total & & 4954 & 929 & 19 \\
\hline
\end{tabular}

*According to InCites Journal Citation Reports (JCR)

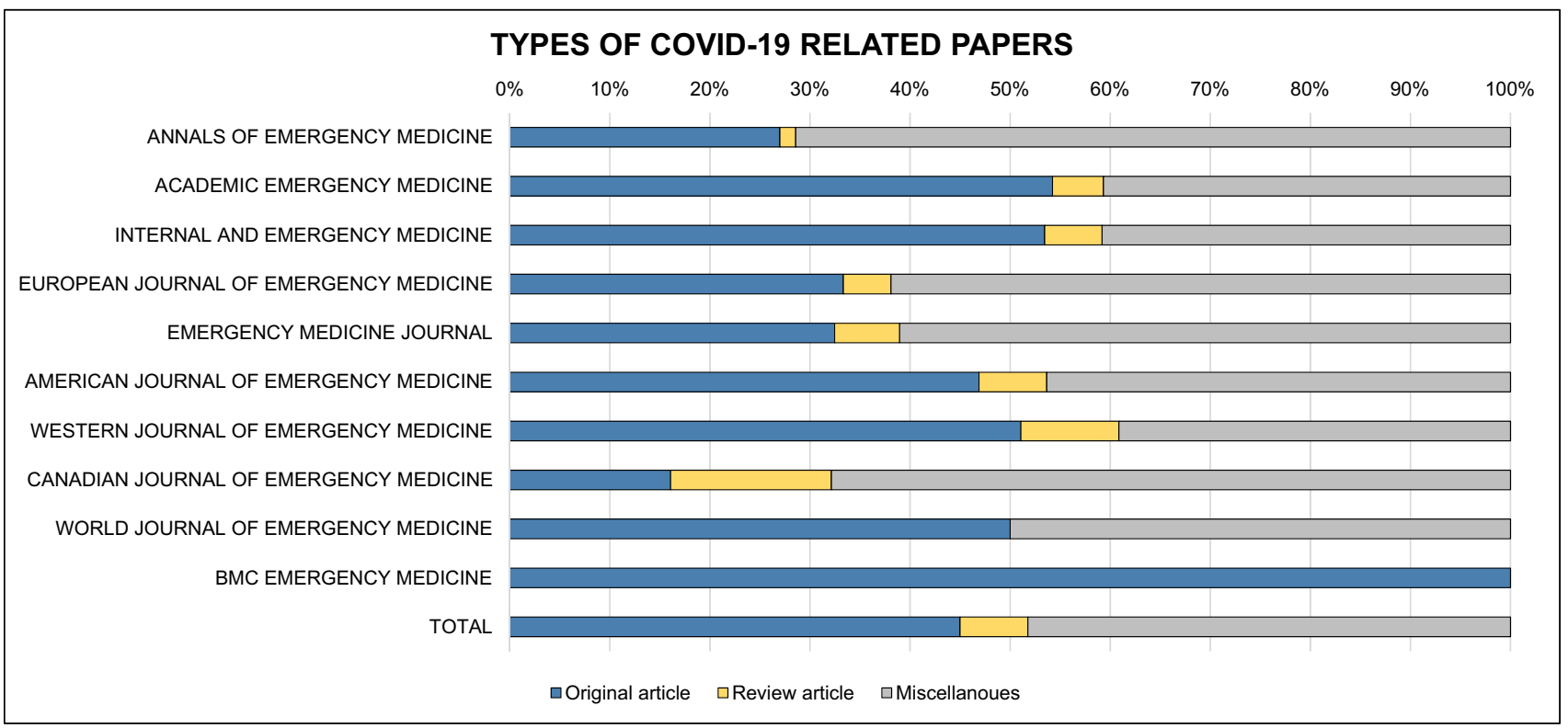

Legend: "original article" includes original articles, research letters and meta-analyses; "review article" includes narrative and systematic reviews, position papers, consensus statements and guidelines; "miscellaneous articles" includes commentaries/editorials, opinion papers, viewpoints, perspectives, case reports, letters and replies

Fig. 1 Different types of COVID-19 related papers among the selected journals: "original papers", "review papers", and "miscellaneous papers" (i.e. commentaries/editorials, opinion papers, viewpoints, perspectives, case reports, letters and replies). "Original article" includes original articles, research letters and meta-analyses;

Medicine to the maximum of $29 \%$ in Internal and Emergency Medicine.

Among the total number of included articles, there were 418 original articles, 63 review articles and 448 papers in the miscellaneous group, corresponding, respectively, to $45 \%, 7 \%$ and $48 \%$ of the total number of COVID-19 related publications (Fig. 1). The most discussed topic among published articles was the impact of COVID-19 on healthcare "review article" includes narrative and systematic reviews, position papers, consensus statements and guidelines; "miscellaneous articles" includes commentaries/editorials, opinion papers, viewpoints, perspectives, case reports, letters and replies

organization that was analyzed in 380 papers $(40.9 \%)$ (Fig. 2, top panel). Specific EM topics such as arrhythmias/ cardiac arrest, ischemic heart disease and thromboembolic events had a relatively limited representation among the selected journals (ranging from 1.9 to $4.4 \%$ ). Since Internal and Emergency Medicine had the highest proportion of articles related to COVID-19 compared to others journal, we performed an additional sub-analysis specifically focused on 
the temporal trends of the main topics discussed in the journal (Fig. 2, bottom panel). After an initial and expected delay of 2 months (January and February 2020) it became clear a substantial rise in COVID-19 related papers focused on the epidemiology, diagnosis and prognosis of the disease (Fig. 2, bottom panel). Additionally, we analyzed the distribution of the articles published within the three sections of the Journal (i.e. Internal Medicine [IM], Emergency Medicine [EM] and Clinical Evidence and Health Technology Assessment [CE]). It was interesting to note that the proportion of the articles published in the specific EM section of the Journal was lower compared to IM and CE sections [25/174 (14.4\%) vs 76/174 (43.6\%) and 73/174 (42.0\%), respectively].

In the present analysis, we aimed to focus on the literary production in the EM field, as primarily involved in the clinical management of pandemic and early impacted by the need of a healthcare reorganization. We selected a subgroup of main EM journals and provided further insights on the contribution of emergency physicians to COVID-19 literature.

During the considered period, the proportion of COVID19 related papers exceeded $15 \%$ of the total publications in most of the Journals. Indeed, it has been reported a significant upsurge in the number of submissions to EM journals after the (World Health Organization) WHO pandemic declaration.

The unexpected face-off with the pandemic has brought healthcare systems on the verge of the collapse, making it necessary to substantially and promptly reorganize healthcare services $[7,8]$. Emergency units have been early reassessed to identify infected patients and select cases deserving hospitalization or more intensive cares [9]. Indeed, emergency physicians promptly published their experiences to share the weakness and strength of local organization, with the aim to facilitate other colleagues in different countries [10]. In accordance with our analysis, a recent bibliometric study also highlighted that the effects of COVID19 on the healthcare system were the most studied topic (222/481, 46.1\%) [11]. Our study actually extends these previous results including a more extended period of time, including the subsequent waves of the pandemic (until May 2021) and offers a broader and updated view of the impact of EM journals to COVID-19 scientific literature.

The importance of rapid and effective communication among EM physicians during the pandemic is reflected by the high percentage of papers published as short publications (e.g. letter, editorial, case reports) and also by the critical role of social media (such as Twitter) in the dissemination of medical information $[12,13]$. As shown by our analysis, the proportion of commentaries/editorials, opinion papers, case reports, letters and replies were at least half of the total publications in the most of Journals analyzed. This may also be a direct effect of the increased workload and stress of EM physicians who were forced to dedicate less time to research activity and data collection [14].

The pressure caused by the pandemic on emergency departments led to an intense reorganization of the hospitals. As reported in our analysis, the need for a rapid healthcare reorganization is clearly reflected by the high percentage of paper COVID-19-related on this issue (up to 64\% in some journals). Indeed, a key element in the management of patients admitted to ED during a pandemic has been the early recognition and immediate isolation of infected patients in preventing infection spreading. In most cases, hospitals needed to create separate ED to threat infected or suspected COVID-19 patients. In the first months of the pandemic, EM journals published several papers reporting local or national experiences of healthcare reorganization. The restless race of the scientific and researcher community has been mainly driven by the urgent need for an effective strategy in facing pandemic spread and was oriented to offer rapid responses to colleagues involved in frontline management of affected patients. The natural consequence has been a marked escalation in journal submissions and an incredible rise in the number of published papers especially focused on the healthcare reorganization [12]. However, collateral effects have been an increased rate of premature publications, requiring corrigendum or retractions, and the shortening and simplification of peer-review processes, resulting in a high rate of inaccuracies, errors or misinterpretation of data [15].

Our study has specific limitations that should be addressed. First, our study is based on the analysis of a sample of EM journals that are not completely representative of all the journals on EM. A specific limitation is also related to papers' categorization since we grouped different types of papers in the "miscellaneous group" to simplify the results, avoiding an excessive fragmentation of the publications categories.

In conclusion, our analysis highlights that EM scientific literature has been significantly involved in the COVID-19 pandemic. We observed a rapid escalation along with time in published reports related to COVID-19, with a marked rise in papers especially focused on healthcare re-organization to face the pandemic. 


\section{Topics in COVID-19 related papers}

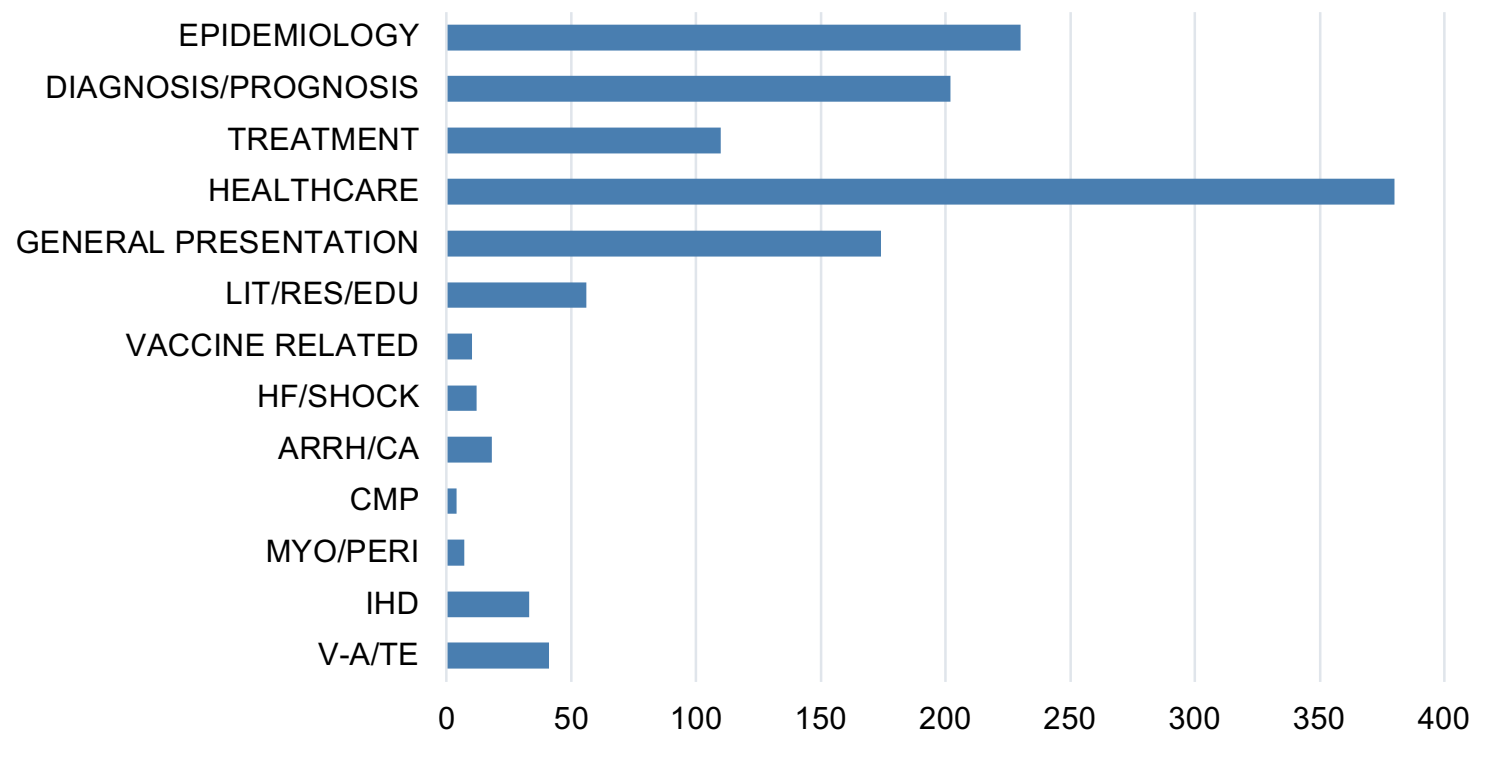

\section{Temporal trends - Internal and Emergency Medicine}

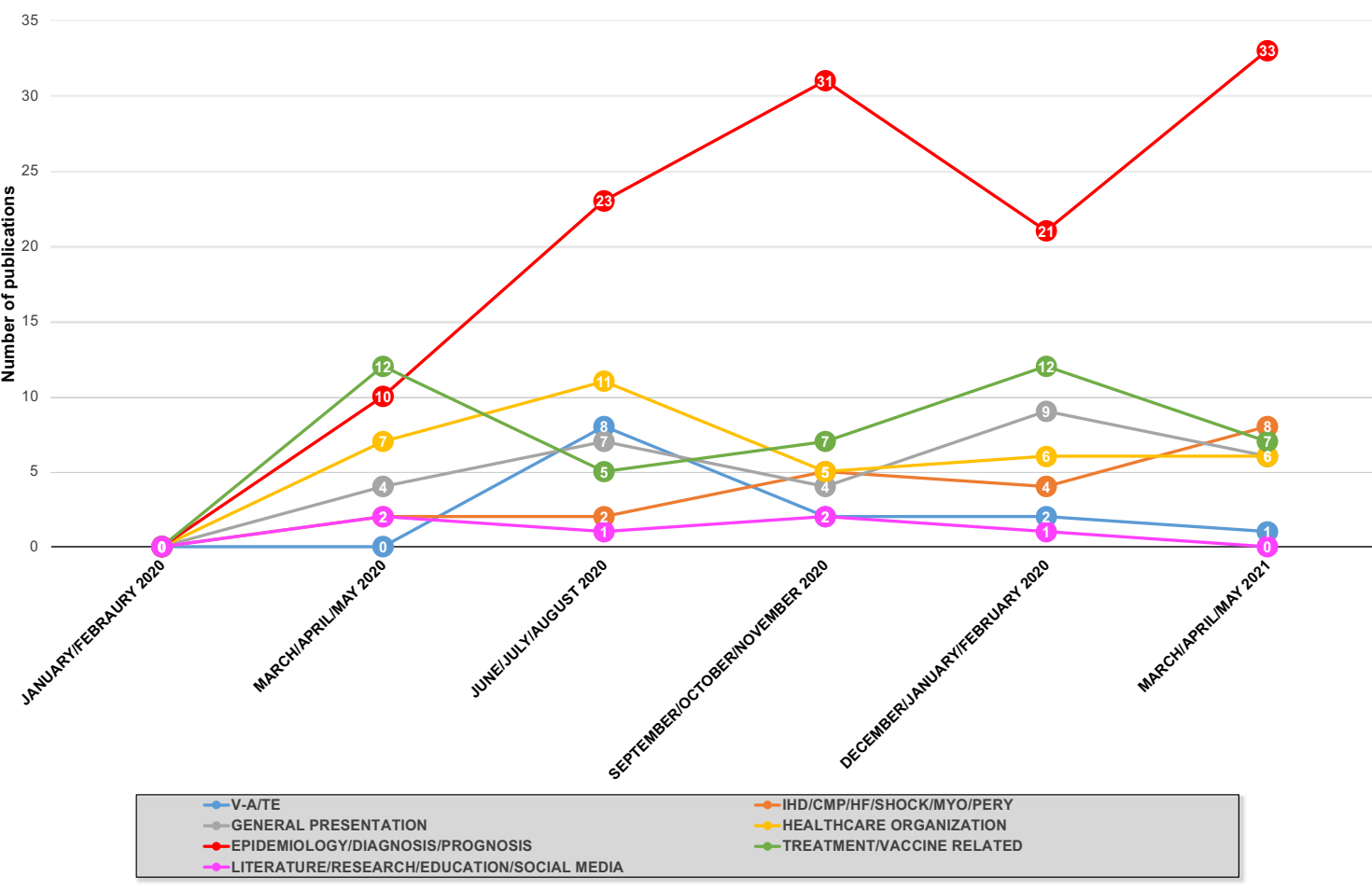

Legend: ARRH/CA, arrhythmias and cardiac arrest; CMP, cardiomyopathies; HEALTHCARE, Healthcare organizations; HF, heart failure; IHD, ischaemic heart disease; LIT/RES/EDU, literature, research, education and social media; MYO/PERI, myocarditis and pericarditis; V-A/TE, venous and arterial thromboembolic manifestation 
4 Fig. 2 Absolute number of the main topics related to COVID-19 as considered by published papers among the ten selected journals. Different topics can be included in a single paper (top panel). Internal and Emergency Medicine temporal trends analysis regarding the main topics COVID-19-related discussed during the study period (bottom panel). ARRH/CA arrhythmias and cardiac arrest, CMP cardiomyopathies, HEALTHCARE Healthcare organizations, $H F$ heart failure, $I H D$ ischaemic heart disease, LIT/RES/EDU literature, research, education and social media, MYO/PERI myocarditis and pericarditis, $V-A / T E$ venous and arterial thromboembolic manifestation

Funding No funding was received for this work.

\section{Declarations}

Conflict of interest The authors report no conflict of interests related to the present article. G Boriani reported small speaker fees from Medtronic, Boston, Boehringer Ingelheim and Bayer outside of the submitted work.

Human and animal rights statement This article does not contain any studies with human participants or animals performed by any of the authors.

Informed consent For this type of study formal consent is not required.

\section{References}

1. Banai A, Taieb P, Furie N, Hochstadt A, Merdler I, Sapir O, Granot Y, Lupu L, Ghantous E, Borohovitz A et al (2021) COVID-19, a tale of two peaks: patients' characteristics, treatments, and clinical outcomes. Intern Emerg Med 16:1629-1639. https://doi.org/10.1007/s11739-021-02711-1

2. Else H (2020) How a torrent of COVID science changed research publishing-in seven charts. Nature 588:553. https://doi.org/10. 1038/d41586-020-03564-y

3. Venturelli A, Vitolo M, Albini A, Boriani G (2021) How did COVID-19 affect medical and cardiology journals? A pandemic in literature. J Cardiovasc Med. https://doi.org/10.2459/JCM.00000 00000001245

4. Boriani G, Vitolo M (2020) COVID-19 pandemic: complex interactions with the arrhythmic profile and the clinical course of patients with cardiovascular disease. Eur Heart J. https://doi.org/ 10.1093/eurheartj/ehaa958
5. Corica B, Romiti GF (2021) Assessing inflammatory status in COVID-19: a role in the pandemic? Intern Emerg Med 16:14231425. https://doi.org/10.1007/s11739-021-02706-y

6. Barakat AF, Shokr M, Ibrahim J, Mandrola J, Elgendy IY (2020) Timeline from receipt to online publication of COVID-19 original research articles. medRxiv. https://doi.org/10.1101/2020.06.22. 20137653

7. Boriani G, Palmisano P, Guerra F, Bertini M, Zanotto G, Lavalle C, Notarstefano P, Accogli M, Bisignani G, Forleo GB et al (2020) Impact of COVID-19 pandemic on the clinical activities related to arrhythmias and electrophysiology in Italy: results of a survey promoted by AIAC (Italian Association of Arrhythmology and Cardiac Pacing). Intern Emerg Med 15:1445-1456. https://doi. org/10.1007/s11739-020-02487-w

8. Campanile A, Verdecchia P, Ravera A, Coiro S, Mattei C, Scavelli F, Bearzot L, Cutolo A, Centola M, Carugo S et al (2021) Intensive cardiac care unit admission trends during the COVID19 outbreak in Italy: a multi-center study. Intern Emerg Med 16:2077-2086. https://doi.org/10.1007/s11739-021-02718-8

9. Castoldi L, Solbiati M, Costantino G, Casiraghi E (2021) Variations in volume of emergency surgeries and emergency department access at a third level hospital in Milan, Lombardy, during the COVID-19 outbreak. BMC Emerg Med 21:59. https://doi.org/ 10.1186/s12873-021-00445-Z

10. Sgura FA, Arrotti S, Cappello CG, Boriani G (2020) Complicated myocardial infarction in a 99-year-old lady in the era of COVID19 pandemic: from the need to rule out coronavirus infection to emergency percutaneous coronary angioplasty. Intern Emerg Med 15:835-839. https://doi.org/10.1007/s11739-020-02362-8

11. Erenler AK, Baydin A (2021) Comparison of performances of top emergency medicine journals in terms of COVID-19 publications in 2020. Am J Emerg Med 46:132-136. https://doi.org/10.1016/j. ajem.2021.03.003

12. Nigrovic LE, Napper T (2021) Marked escalation in journal submissions during COVID-19 pandemic. Ann Emerg Med 77:130 131. https://doi.org/10.1016/j.annemergmed.2020.07.003

13. Rosenberg H, Syed S, Rezaie S (2020) The twitter pandemic: the critical role of twitter in the dissemination of medical information and misinformation during the COVID-19 pandemic. CJEM 22:418-421. https://doi.org/10.1017/cem.2020.361

14. Vanni S, Nazerian P, Bini G (2020) From the quarantine diary of an emergency physician: the coronavirus and the dysautonomic storm. Intern Emerg Med 15:1361-1364. https://doi.org/10.1007/ s11739-020-02352-w

15. The Lancet Global Health (2020) Publishing in the time of COVID-19. Lancet Glob Health 8:860. https://doi.org/10.1016/ S2214-109X(20)30260-6

Publisher's Note Springer Nature remains neutral with regard to jurisdictional claims in published maps and institutional affiliations. 